\title{
Batch crystallisation methods for serial crystallography experiments
}

\author{
John H. Beale, May E. Sharpe \\ Paul Scherrer Institut, Villigen-PSI, Switzerland \\ john.beale@psi.ch
}

The applications and potential advantages of serial crystallography, at both synchrotron and XFEL light sources, are growing. Despite advances in delivery methods, the sample volumes of micro-crystals required for serial crystallography, particularly time-resolved experiments, are still demanding. Batch crystallisation methods are the primary means in crystallographer's toolbox to create these samples. However, the process to convert single crystals grown by vapour diffusion to large volumes $(>100 \mu \mathrm{L})$ of micro-crystalline slurry can be exceptionally challenging. Here we present a strategy to perform this translation and it is divided into three stages: (1) optimising crystal morphology, (2) transitioning to batch, and (3) scaling. Given the variation of protein crystallisation, we hope that this protocol can act as a useful framework when attempting the conversion from vapour diffusion to batch. Tips and tricks will also be presented that may also be useful. Ultimately, we hope that this methodology improves the samples used for serial crystallographic studies and that this also improves the quality of acquired data.

Keywords: Serial crystallography, batch crystallization, micro-crystallization, XFEL, phase diagram 